\title{
Mechanical Properties of Silicon Nitride Using RUS \& C-Sphere
}

\section{Methodology}

\author{
Mark Hadfield ${ }^{1, a}$, Wei Wang ${ }^{2, b}$ and Andrew Wereszczak ${ }^{3, c}$ \\ ${ }^{1}$ School of Design, Engineering and Computing, Bournemouth University, Poole, BH12 5BB, UK \\ ${ }^{2}$ School of Design, Engineering and Computing, Bournemouth University, Poole, BH12 5BB, UK \\ ${ }^{3}$ Materials Science and Technology Division, Oak Ridge National Laboratory, Oak Ridge, TN \\ 37831, United States
}

amhadfield@bournemouth.ac.uk, 'wei.wang2@rolls-royce.com, cwereszczakaa@ornl.gov

Keywords: RUS, C-Sphere, Surface Strength, Elastic Property, Silicon Nitride

\begin{abstract}
Silicon nitride is a type of engineering ceramic which has been used in ball bearing and other rolling contact applications owing to its good fatigue life, high temperature strength and tribological performance. In this paper, the mechanical properties of Hot Isostatically Pressed (HIPed) and Sintered and Reaction Bonded Silion Nitride (SRBSN) have been studied. The elastic modulus and poisson's ratio of three types of commerical grade HIPed silicon nitride, and ground SRBSN with three surface condidtions were measured using a Resonance Ultrasound Spectroscopy (RUS). The RUS measurement reveals the variation of elastic properties across different types of HIPed silicon nitride specimens. The surface strength of silicon nitride are studied using a C-sphere specimen, and the results show that SRBSN with three different surface finishing conditions show varied surface strength. The RUS and C-sphere techniques can potentially be used to sample the quality and consistency of ball bearing elements.
\end{abstract}

\section{Introduction}

Silicon nitrde has been developed into applications in roller/ball bearings [1-3] and various rolling contact applications in diesel engine. Understanding of rolling contact fatigue (RCF) of silicon nitride is important to assess the reliability of these silicon nitride components. RCF can be evaluated through either full scale bearing testing or laboratory bench testing. Full scale testing is complex to setup, so in practice tribotesters are used to study RCF. Evaluation of the mechanical properties of silicon nitride, such as the elastic property and surface strength is important to the understanding of the RCF performance of silicon nitride prior to bench testing [4]. These evaluations can help to screen out the weak material before the start of lengthy RCF testing.

\section{RUS Measurements}

The RUS measurement is a non-destructive technique to accurately measure the elastic property of silicon nitride. The mechanical resonance of a component is a funciton of its shape, density, and elastic properties. The RUS measurement is essentially based on this principle such that a measurement of the resonant frequencies provides a unique chacteristics to the component. A resonance spectrum is generated by sweeping the frequency of an ultrasound signal applied to the components and by detecting its resulting resonance frequencies. A typical silicon nitride RUS spectrum is shown in Fig 1. Changes in either the shape, density or elastic properties, or introduction of defects, lead to a variation of this characteristics.

The measurement is carried out on a Resonance Ultrasound Spectroscope. The silicon nitride specimen is secured by three acoustic probes, which contains one acoustic generator and two acoustic receivers. All three probes are connected to an acoustic processor for data analysis, and the schematic of the measurement setup is shown in Fig 2. As the silicon nitride ball specimen 
geometry can be accurately controlled, the spectrum change due to geometry variation is minimal. Therefore the density and elastic property of the specimen dictates the resonant spectrum.

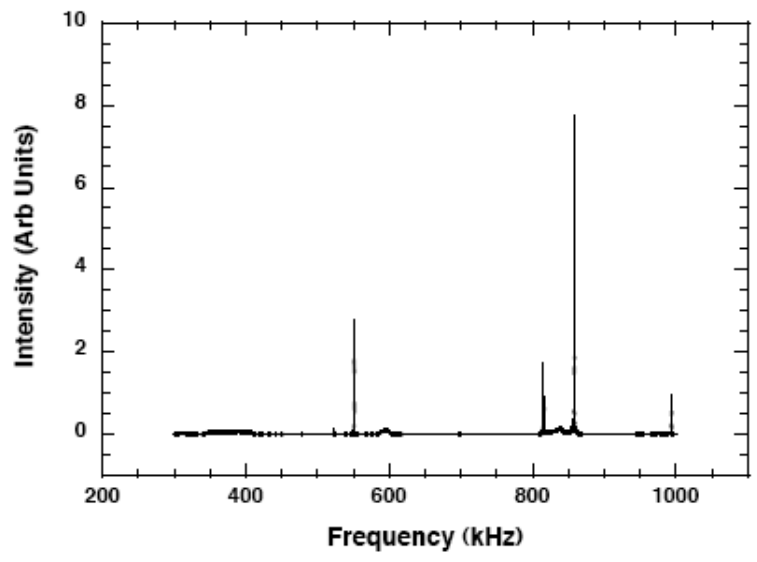

Figure 1: Silicon nitride RUS spectrum

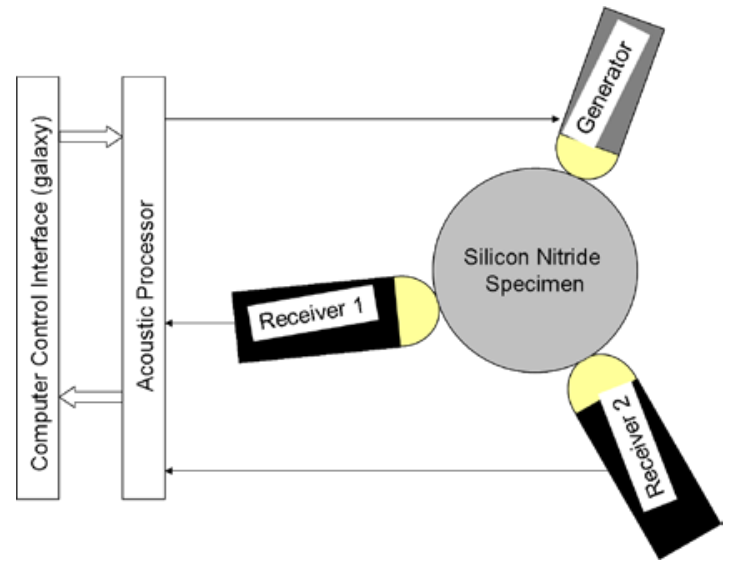

Figure 2: Schematic of RUS measurement

Six batches of silicon nitride specimens with 20 in each batch are examined using RUS measurement. Fig 3 shows the results of three batches of specimens with coarse, fine and conventional finishing conditions machined from the same SN-A SRBSN material. The results indicate that the difference in machining procedures doesn't significantly affect the elastic property of silicon nitride. The exceptions in the data shown in the circle are largely due to the mass/density variation of the specimens, which is possibly a result of inhomogeneity or porosity in the primary processing. RUS measurement can screen out these specimens with defects and exclude them from further RCF testing. It also demonstrate that the instrinic property of SN-A is poor, and potentially not suitable for rolling contact applications which demands reliability, as the failure can initiate from the inhomogeneity.

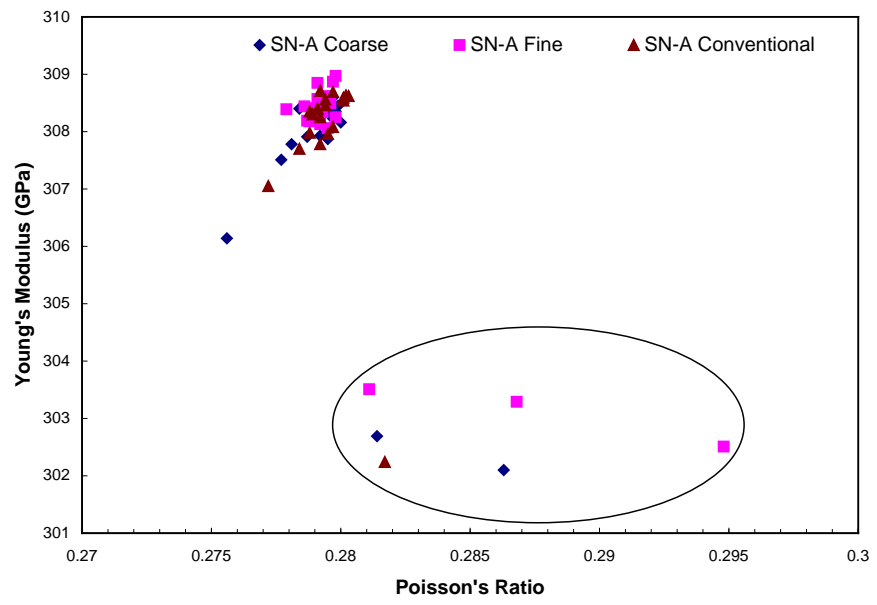

Figure 3: 3 batches of SN-A RUS result

Fig 4 shows the results of the other three batches of silicon nitride material representing Toshiba TSN03NH, NBD200 and SN101C respectively. These three Silicon Nitride are all processed through HIPing process, but the difference on additives and processing parameters gives them different elastic properties. The normal distribution of the measured young's modulus in Fig 5 shows that NBD200 material has a higher Young's modulus than TSN03NH and SN101C material. TSN03NH material has a narrower range of distribution, which indicates that potentially TSN03NH has better consistency in material processing. 


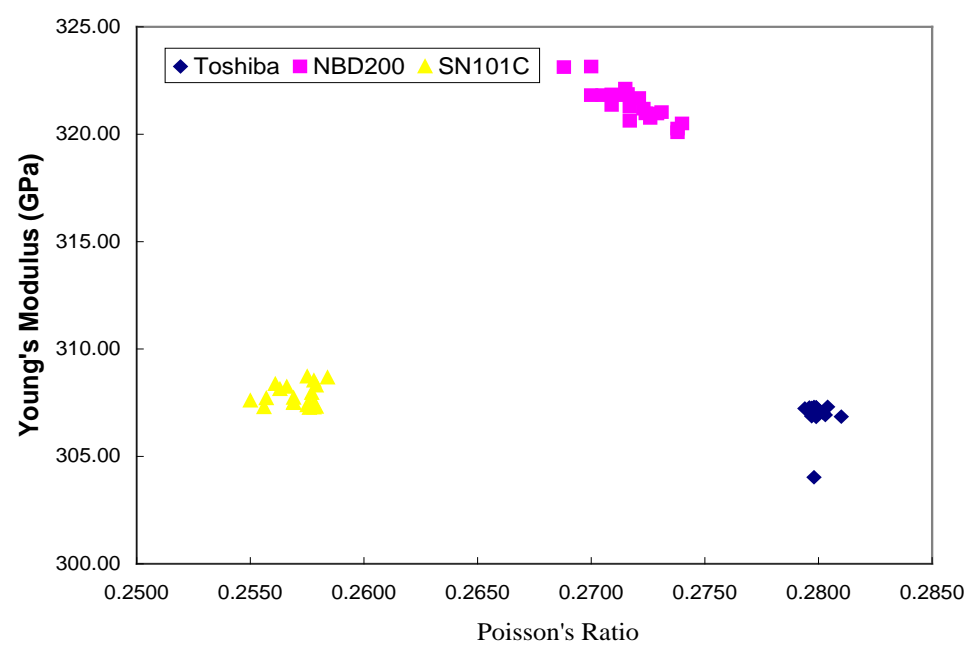

Figure 4: Elastic properties of three HIPed Silicon Nitrides.

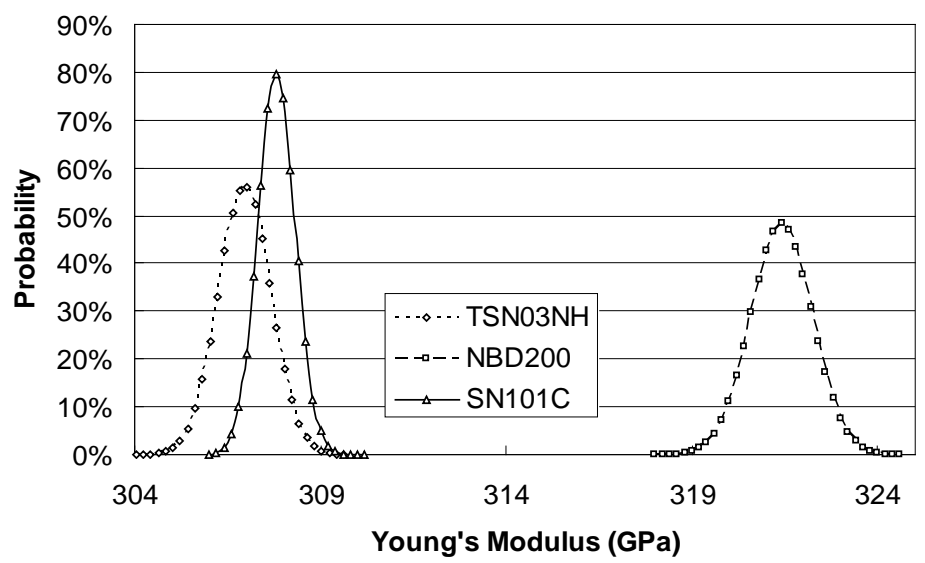

Figure 5: The normal distribution of elastic properties measured on three commerical grade HIPed Silicon Nitrides

The variation in Young's modulus can result in a change in Hertzian contact stress when the silicon nitride balls are subjected to RCF test contact geometry. Without accurate Young's modulus measurement and Poisson's ratio's measurement, the generic values of $315 \mathrm{GPa}$ and 0.280 are normally used when calculating the Hertzian contact stress. Fig 6 shows the calculated Hertzian stress as a funtion of Young's modulus. The contact stress using measured elastic properties varies from batch to batch, and it also varies within the same batch of specimens. RUS measurement is simple technique to ensure that accurate elastic properties of candidate silicon nitride is used to calculate the Hertzian stress.

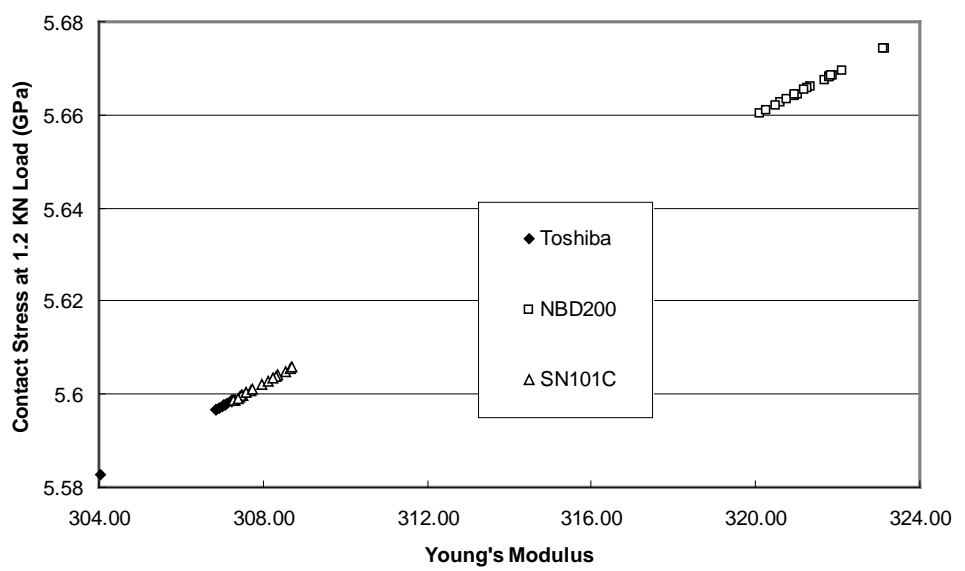

Figure 6: The effect of Young's modulus on Hertzian contact stress 


\section{C-sphere Strength}

The C-sphere [5] technique was derived from the C-ring test which was traditionally used to examine the strength and subsurface damage of tubular components [6]. Grinding of the slot was performed in a two-step process using a diamond plated grinding wheel (5 in. dia. $\mathrm{x} 0.25 \mathrm{in}$. thick with a full $0.125 \mathrm{in}$. radius or $127 \mathrm{~mm}$ dia. x $6.35 \mathrm{~mm}$ thick x $3.175 \mathrm{~mm} \mathrm{R}$ ) for the final grinding. Csphere flexure specimens were monotonically and compressively loaded to failure, as shown in Fig 7 using an electromechanical universal testing machine at a crosshead displacement rate of 0.5 $\mathrm{mm} / \mathrm{min}$.

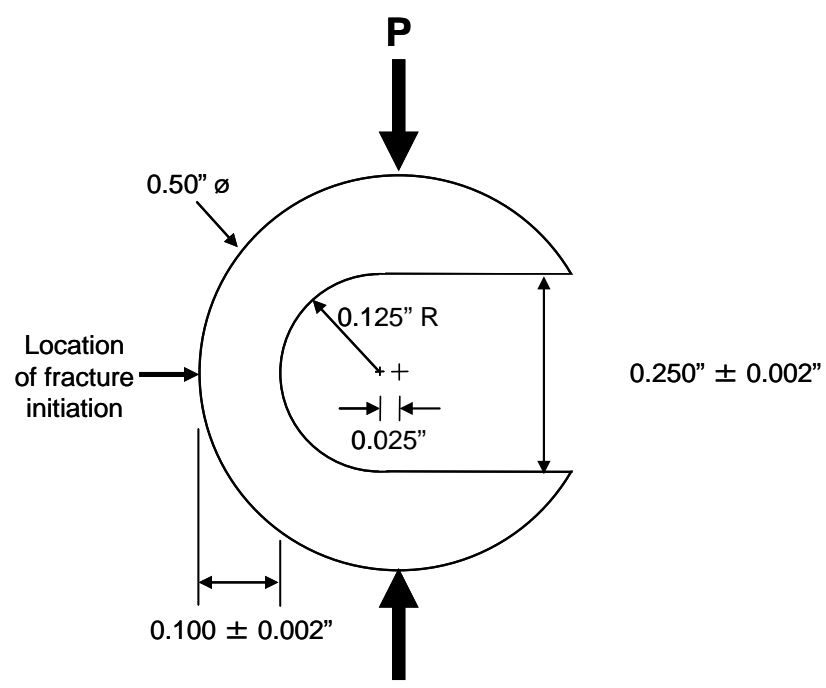

Figure 7: C-sphere flexure strength loading schematic.

Three batches (5 in each batch) of Ceralloy SRBSN silicon nitride C-sphere specimens were tested and each batch of specimens has a different surface finishing condition, varying from coarse to fine and RCF-conventional (the best finish possible). The typical fracture surfaces are shown in Fig 8 and the surface strength result is shown in Fig 9.

The RCF-Conventional ceralloy specimens has a superior effective strength compared with fine and coarse ceralloy specimens. It is $\sim 12 \%$ and $\sim 13 \%$ better than fine and coarse specimens respectively. With a fracture toughess of $6 \mathrm{MPa} \sqrt{\mathrm{m}}$ and approximate crack geometry factor 1.5 , the effective Griffith flaw size of coarse, fine and RCF-Conventionally finished specimens are 25.3, 24.8 and 19.9 microns respectively.

The C-sphere strength result indicates that the more agressively machined surface conditions has larger machining induced defects, which weaken the surface strength. The surface integrity is a good indicator of potential RCF performance, therefore C-sphere can be used as technique to compare the potential RCF performance of candidate specimens.
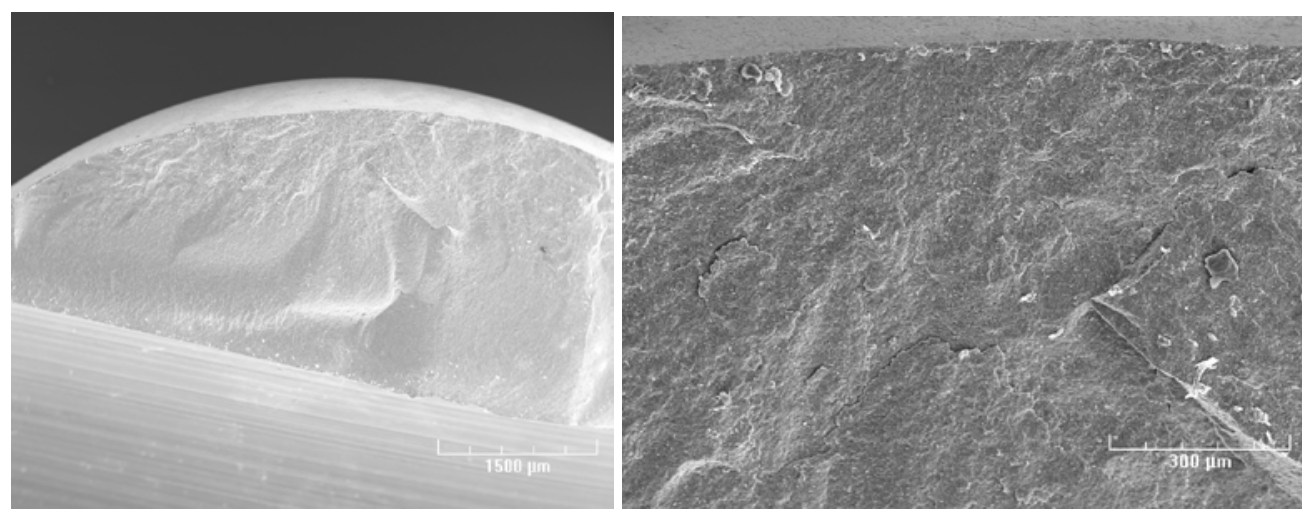

Figure 8: The typical fracture 


\section{Ceralloy 147-31N C-Sphere Strength Comparison 0.50 " dia $(12.7 \mathrm{~mm})$}

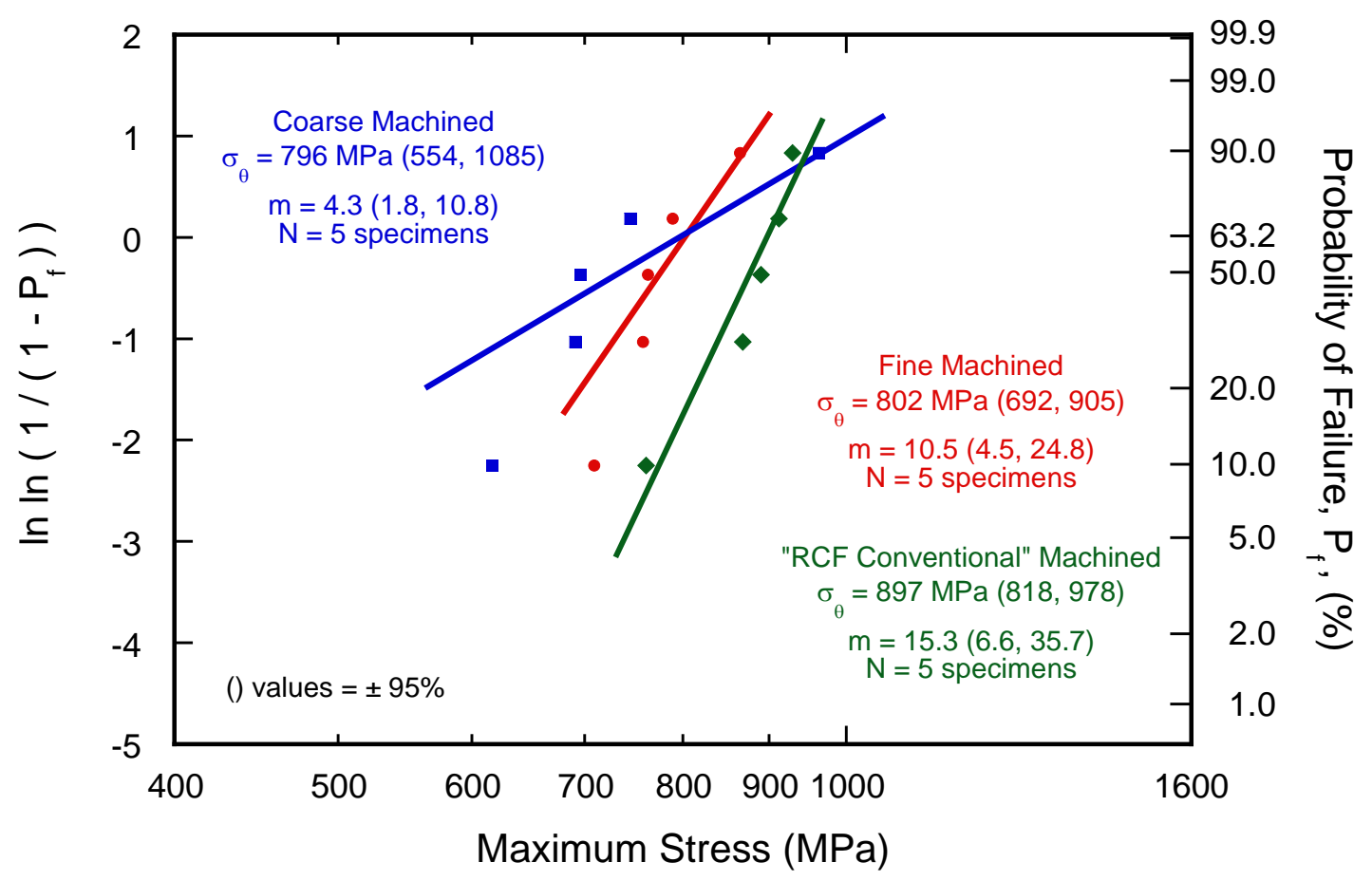

Figure 9: C-Sphere Weibull Strength Distribution of Ceralloy Specimens

\section{Concluding Remarks}

RUS measurement is simple empirical technique which can potentially be used for the screening of material porosity and inhomogeneity. The C-sphere strength is an indicator of surface integrity. Using C-sphere strength, it can compare the potential RCF performance of the candidate specimens. The RUS and C-sphere techniques can potentially be used to sample the quality and consistency of ball bearing elements.

\section{References}

[1] L. Wang, R. W. Snidle, L. Gu, Wear 246 (2000) 159-173.

[2] R. N. Katz, International Journal of High Technology Ceramics 1 (1985) 69-79.

[3] L. Y. Chao, D. K. Shetty, Journal of Materials Education 17 (1995) 245-303.

[4] W. Wang, A. A. Wereszczak, M. Hadfield, Ceramics International 35 (2009) 3339-3346.

[5] A. A. Wereszczak, T. P. Kirkland, O. Jadaan, Journal of American Ceramic Society 90 (2007) 1843-1849.

[6] A. A. Wereszczak, R. J. Caspe, J. J. Swab, S. F. Duffy, E. H. Baker, C-ring strength of advanced monolithic ceramics, in: 27th International Cocoa Beach Conference on Advanced Ceramics and Composites: B, vol 24, 2003, pp. 483-490. 